\title{
Particulate Binary Mixtures: Dependence of Packing Porosity on Particle Size Ratio
}

\author{
Ricardo P. Dias, ${ }^{\dagger}$ José A. Teixeira, ${ }^{\ddagger}$ Manuel G. Mota, ${ }^{*, *}$ and Alexander I. Yelshin \\ Instituto Politécnico de Bragança, Esc. de Tecnologia e Gestão, Campus de Sta. Apolónia, Ap. 1038, \\ 5301-854 Bragança, Portugal, and Centro de Eng. Biológica, University of Minho, Campus de Gualtar, \\ 4710-057 Braga, Portugal
}

\begin{abstract}
Binary mixtures with significant size ratios are scarcely studied. Yet, contaminants of chromatographic columns or ion-exchange resins have size ratios of $\delta<0.1$. Binary mixtures of glass beads with $\delta=0.1-0.0375$ were used experimentally to measure packing porosity. Simultaneously, a significant number of published data was analyzed. A linear mixing model was adopted to predict the porosity of each particle fraction in the binary mixture. Deviations from the model may be caused by wedging of small particles between the large ones. Large particles may disturb the porous medium properties by inducing a wall effect over the small particles. Wedging analysis led to the conclusion that, for $\delta<0.01$, its effect is insignificant. The wall effect yields an additional void around the large particles as long as $\delta>0.0035$. For $\delta<0.0035$, the small particles form a monosized dense packing and both wedging and wall effects become negligible.
\end{abstract}

\section{Introduction}

Models of fluid flow or mass transfer in porous media need to establish relationships of packed-bed porosity with permeability or diffusivity., ${ }^{1,2}$ In solid-liquid separation, for instance, cakes formed in filtration are often represented by their composition in coarse and fine particles.

Depending on packing conditions, it is possible to obtain either a regular packing of monosized spheres or a random packing. ${ }^{3,4}$ Regular packing beds are associated with a fixed coordination number $\left(N_{\mathrm{c}}\right)$, which is defined as the number of contacts of each sphere with neighboring spheres. ${ }^{3,5}$

The packing density becomes less predictable for a random arrangement of spheres, where the coordination number is defined as the average value for all particles. The same procedure used to obtain a homogeneous monosized particle packing may produce a packing of different porosity when particles with different sizes ${ }^{6,7}$ are used. Even when only two sphere sizes are used, porosity and permeability will be dependent on the size ratio between spheres and on the volume fraction of each type of sphere.

To clarify the relationship between packing porosity and small particle size ratio $(\delta)$, binary mixtures of spheres of different size will be investigated and analyzed in the present work.

\section{Theoretical Background}

Models of mixed particle-bed porosity versus the fractional content and particle size ratio have been

* To whom correspondence should be addressed. E-mail address: MMota@reitoria.uminho.pt.

† Instituto Politécnico de Bragança.

$\doteqdot$ University of Minho. described in many publications. ${ }^{4,8-19}$ However, deviations of experimental data from theoretical prediction induce the development of research to seek the causes of these deviations.

A linear mixture model, which is applied for binary packing when the ratio of small particle size $(d)$ to large particle size $(D)$ is $\delta=d / D \rightarrow 0$, is used in numerous works. ${ }^{8-13,17,20}$ The overall porosity $(\epsilon)$ is presented in the form of the product of fractional porosities: $\epsilon_{D}=$ $\epsilon_{D}\left(x_{D}\right)$ and $\epsilon_{d}=\epsilon_{d}\left(x_{D}\right)$, where $\epsilon_{D}$ is the void fraction of large particles in the total volume of the mixture and $\epsilon_{d}$ is the specific void fraction of small particles in the remaining void volume of the mixture. Because the overall volume of solids in the mixture $(1-\epsilon)$ is the sum of the volumes of large particles $\left(1-\epsilon_{D}\right)$ and those of small particles $\left(\left(1-\epsilon_{d}\right) \epsilon_{D}\right)$, the porosity of the mixture becomes ${ }^{2,21}$

$$
\epsilon=\epsilon_{d} \epsilon_{D}
$$

Both $\epsilon_{d}$ and $\epsilon_{D}$ are functions of $x_{D}$ and are dependent not only on $\epsilon_{d}, \epsilon_{D}^{0}$, and $\delta$, but also physical effects in the mixture, namely, friction forces and the packing preparation method.

The fractional porosity of the large particle fraction is defined as

$$
\epsilon_{D}=1-(1-\epsilon) x_{D} \quad\left(\text { for } \epsilon_{D} \in\left[1, \epsilon_{D}^{0}\right]\right)
$$

The boundary conditions are as follows: when $x_{D}=0$, $\epsilon_{D}=1.0 ;$ if $x_{D}=1.0$, then $\epsilon=\epsilon_{D} \equiv \epsilon_{D}^{0}$.

The fractional porosity of the small-size particle fraction may be obtained from eq 1 : 


$$
\epsilon_{d}=\frac{\epsilon}{\epsilon_{D}}=\frac{\epsilon}{1-(1-\epsilon) x_{D}} \quad\left(\text { for } \epsilon_{d} \in\left[1, \epsilon_{d}^{0}\right]\right)
$$

The boundary conditions are as follows: if $x_{D}=0$, then $\epsilon=\epsilon_{d} \equiv \epsilon_{d}^{0}$; if $x_{D}=1.0$, then $\epsilon_{d}=1.0$. Using eqs 1 and 2 , the following relation can be derived for the largeparticle volume fraction:

$$
x_{D}=\frac{1-\epsilon_{D}}{1-\epsilon}=\frac{1-\epsilon_{D}}{1-\epsilon_{d} \epsilon_{D}}
$$

Now it is possible to derive formulas for the overall porosity (eqs 5 and 6) and for fractional porosities (eqs 7 and 8):

$$
\begin{gathered}
\epsilon=\epsilon_{D} \epsilon_{d}=\frac{\epsilon_{d}\left(1-x_{D}\right)}{1-\epsilon_{d} \epsilon_{D}} \\
\epsilon=1-\frac{1-\epsilon_{D}}{x_{D}} \\
\epsilon_{d}=\frac{\epsilon_{D}+x_{D}-1}{\epsilon_{D} x_{D}} \\
\epsilon_{D}=\frac{1-x_{D}}{1-\epsilon_{d} x_{D}}
\end{gathered}
$$

Under the conditions $\epsilon_{D}=\epsilon_{D}^{0}$ and $\epsilon_{d}=\epsilon_{d}^{0}$, the system of eqs $4-6$ becomes

$$
\begin{gathered}
\epsilon=\epsilon_{d}^{0}\left(\frac{1-x_{D}}{1-x_{D} \epsilon_{d}^{0}}\right) \quad\left(\text { for } x_{D} \in\left[0, x_{D, \text { min }}\right]\right) \\
\epsilon=1-\frac{1-\epsilon_{D}^{0}}{x_{D}} \quad\left(\text { for } x_{D} \in\left[x_{D, \text { min }}, 1\right]\right)
\end{gathered}
$$

with a minimum porosity of $\epsilon_{\min }=\epsilon_{d}^{0} \epsilon_{D}^{0}$ that corresponds, at large-sized particle volume fractions, to

$$
x_{D, \min }=\frac{1-\epsilon_{D}^{0}}{1-\epsilon_{d}^{0} \epsilon_{D}^{0}}
$$

This system corresponds to the classic linear mixing model, which has been developed by many authors. ${ }^{8-10,13,17,22}$ Equations 9 and 10 represent the two branches of the model, as depicted in Figure 1.

According to the linear model, the dependence of $\epsilon$ on $x_{D}$ is represented by two equations. Equation 9 represents the dependence of $\epsilon$ on $x_{D}$ for mixtures enriched with small particles; for clusters of small particles, the porosity is assumed to be the same as the porosity $\epsilon_{d}^{0}$ of a monosized packing of small particles. Equation 10 represents the dependence for mixtures enriched with large particles. The intersection point of the two functions $\left(x_{D}=x_{D, \min }\right)$ corresponds to a mixture where the amount of large particles is enough to build up a skeleton in the packing.

Transition from the model to the real binary packing system, when the particle size ratio $\delta=d / D$ is considered as a variable, requires the use of some correction functions, because of a variety of possible particle arrangements. Correction functions appear as a result of curve-fitting procedures. ${ }^{2,8,10,17,20,23-31}$

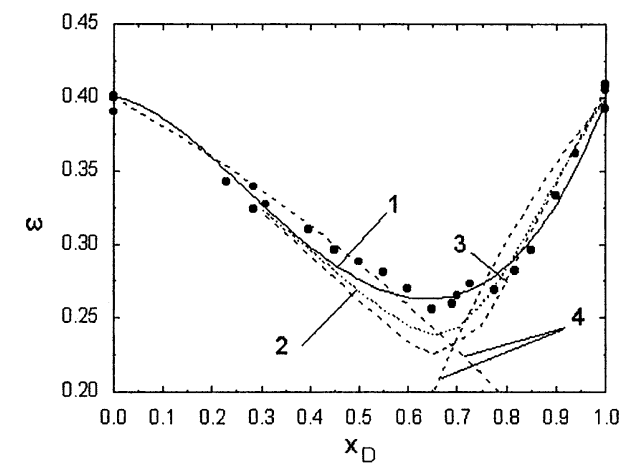

(a)

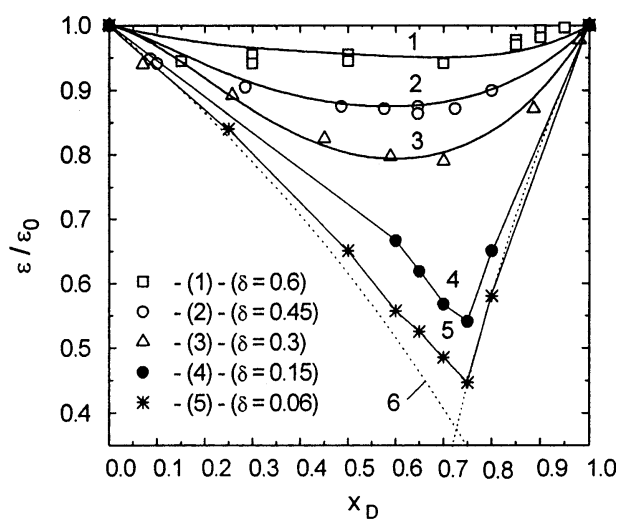

(b)

Figure 1. (a) Comparison of the dependence $\epsilon$ vs $x_{D}$ obtained with different correction function models: data from Mota et al. ${ }^{2}$ (curve 1), data from Caglioti et al. ${ }^{32}$ (curve 2), data from Finkers and Hoffmann $^{36}$ (curve 3), and data from Abe and Hirosue 22 (curve 4). (b) Normalized porosity $\epsilon / \epsilon_{0}$ for data sets of Yu et al. ${ }^{13}$ Curves $1-3$ correspond to the model of Mota et al. ${ }^{2}$ Curve 6 is the boundary limit at $\delta \rightarrow 0$, eqs 1 and 2 .

The particle composition, size ratio, and the packing method affect the properties of mixed beds. ${ }^{2,12,25,26}$ There are, in the literature, several examples of reliable packing procedures, such as (i) mixing in a cylindrical vessel rotating about its axis and rocking around the vertical axis; ${ }^{27}$ (ii) blending the desired proportions in an agitated flask; ${ }^{7}$ (iii) adding premixed particles into a vessel with water; ${ }^{19}$ (iv) portion mixing and wholebed packing procedures; ${ }^{28}$ (v) different variants of shaking a vessel in a circular, and end-over-end fashion, tipped or slowly rotated about its axis, ${ }^{29}$ etc.

A packing procedure may result in a stable homogeneous packing or may cause a segregation effect. ${ }^{30-33}$ All these facts explain why several authors have incorporated correction functions into eqs 1 and 2 in different forms. $.8,11,22,34$

When the particle size ratio is in the range of moderate values, viz, $0.1<\delta<1.0$, the interaction between fractions becomes more pronounced. By analyzing the random packing structure, $\mathrm{Yu}$ and Standish ${ }^{17}$ found that, depending on the value of $\delta$, two packing mechanisms are involved. One is a filling mechanism and the other is an occupation (or mixing) mechanism that acts between particles with small size differences. Based on this approach, the linear-mixture packing model was developed with the incorporation of interaction functions, generally represented by experimentally determined polynomial or power-law functions. ${ }^{13,35,36}$

This brief outline leads shows that, generally, two types of model dependence of $\epsilon$ on $x_{D}$ are proposed: the discontinuity model, when the minimum porosity $\epsilon_{\min }$ 


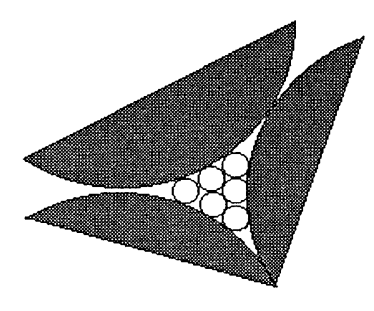

(a)

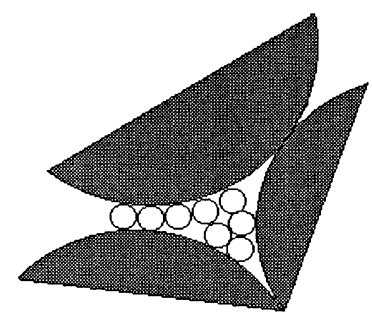

(b)
Figure 2. Schematic representation of the arrangement of small particles between large particles: (a) small particles fill the free space between skeleton particles. (b) small particles wedge in the skeleton of large particles.

is obtained by the intersection of eqs 9 and 10, and the continuity model, with a smooth porosity transition in the region of $\epsilon_{\min }$.

The choice of a continuity or discontinuity model will result in a smooth or broken curve with an intersection point in the region of minimum porosity (see Figure 1). In Figure 1a, the experimentally determined porosity of a binary glass bead mixture of $D / d=10.22$ (indicated by the data points, which were taken from Mota et al. ${ }^{2}$ ) is shown, together with the calculated porosity using different correction functions. ${ }^{2,22,35,36}$

In Figure 1b, a normalized porosity $\left(\epsilon / \epsilon_{0}\right)$ is shown for data sets of Yu et al., ${ }^{13}$ where $\epsilon_{0}=\epsilon_{D}^{0}=\epsilon_{d}^{0}$ are values of $\epsilon_{0}$ that correspond to those referenced in the quoted literature. It can be seen that, with decreasing $\delta$, the smooth transition of minimum porosity (curves 1-3) may become an intersection point (data sets 4 and 5), because the filling mechanism of packing becomes predominant. Note that the left part of $\epsilon\left(x_{D}\right)$ deviates significantly from the theoretical expectation; this fact was observed in numerous publications, $8,10,12$ and several authors explain the deviation with the occurrence of an interparticle wall effect.

The major problem that must be overcome in the attempts to obtain a generalized porosity model of granular beds is the change of the porous system internal scale ${ }^{37,38}$ in the direction of $\delta$ from 1.0 to 0 . Two scale effects are reported: (i) the porous media properties change whenever the small-particle size ratio $\delta$ becomes moderate, and (ii) the porosity fluctuation is due to a wall effect, which is estimated to cause disturbances at a distance of 4-5 sphere diameters away from the wall. ${ }^{39}$ The wall-effect concept in the binary packing was discussed in numerous publications; $9,12,40,41$ however, the effect of each particle fraction on the overall porosity $\epsilon$ has not been investigated very much, especially for $\delta<0.1$.

The linear model of the binary mixture will be used below to analyze the influence of each particle fraction on $\epsilon$ over the entire range of $x_{D}$ values, by means of a fractional porosity approach developed in previous research. ${ }^{2,20,21,42}$ As was mentioned, the intersection point of the two branches corresponds to a mixture where the amount of large particles $\left(x_{D}=x_{D \text {,min }}\right)$ is large enough to build up the supporting skeleton for the smaller particles. This implies that a sequential mixing procedure is used. In turn, when a mixing procedure is applied to the entire binary mixture, another scenario is possible. Small particles are then able to wedge in the skeleton of large particles, resulting in an increase in fractional porosities (see Figure 2). In this case, the overall porosity would be higher than the estimated value by $\epsilon_{\min }=\epsilon_{d}^{0} \epsilon_{D}^{0}$. A good mixing is expectable for
Table 1. Effect of Mixing Procedure on Bed Porosity

\begin{tabular}{ccc}
\hline & \multicolumn{2}{c}{ Value } \\
\cline { 2 - 3 } porosity & nonagitated mixture & agitated mixture \\
\hline$\epsilon_{D}$ & 0.444 & 0.483 \\
$\epsilon_{d}$ & 0.586 & 0.65 \\
$\epsilon$ & 0.26 & 0.314
\end{tabular}

moderate values of $\delta$. Mixing quality decreases for $\delta \ll$ 1.0 (see Figure 1b).

Wall and wedging effects have not yet been studied in detail for small size ratios. This is rather important, for instance, to predict behavior of contaminants in ionexchange resins. The correct representation of the porosity in this range will give more-accurate predictions for the packing permeability. ${ }^{2}$

As was mentioned previously, depending on $\delta$, two packing mechanisms are involved in packed-bed structure formation: ${ }^{17}$ a filling mechanism and an occupation (or mixing) mechanism that acts between particles with small differences in size. These two mechanisms act in such a way that three different situations may occur at moderate $\delta$ values:

(1) For mixtures enriched with small particles, where $x_{D}<0.65$, the amount of large particles is not enough to build up a support skeleton inside the mixture. The mixing procedure does not affect porosity significantly.

(2) The region of minimum porosity, $0.65<x_{D}<0.75$, corresponds to "flooded" beds. Beds can undergo rearrangements, depending on the mixing procedure. When the mixing is done by particle displacement and rotation, small particles are able to wedge between large particles of the skeleton and the overall bed porosity may increase.

(3) Mixtures with a high fraction of large particles, $x_{D}>0.75$. In this case, the formation of the large particle skeleton is easily observed when the particle size ratio is $\delta<0.2$. The smaller particles fill the skeleton void, and beds with stable porosity are obtained.

The small-particle wedging phenomenon is illustrated with data displayed in Table 1 . These experimental data for $D / d=10.22(\delta=0.098)$ and $x_{D}=0.75$ were obtained during tests on packing procedures used in the previous work of Mota et al. ${ }^{2}$ Nonagitated mixtures were simply tapped. As may be observed, the overall porosity, as well as $\epsilon_{D}$ and $\epsilon_{d}$, increased in agitated mixtures when small particles wedged into the large-particle skeleton.

This example shows that, in the region around minimum porosity, the instability of small and large particle arrangement may be a reason for the appearance of a diversity of binary packing arrangements and for the different $\epsilon$ curves reported in the literature.

When the ratio $\delta$ is small enough to allow small particles to penetrate freely into the skeleton void, the skeleton is kept fixed, down to $x_{D \text {,min. }}$. However, when the volume fraction of the large particle $x_{D}$ is below the critical value $x_{D \text {, min }}$, different packing mechanisms begin to work. There are three possibilities: (i) a uniform distribution of large particles in the mixture volume, (ii) the skeleton remains a loose packing until it reaches a stable condition, and (iii) large particles spread throughout the mixture in the form of clusters. With all the implications herewith discussed, experimentation, modeling, and detailed analysis will be performed in the following discussions.

\section{Materials and Methods}

Binary mixtures of glass beads were used in all experiments included in the present work. Beads with 


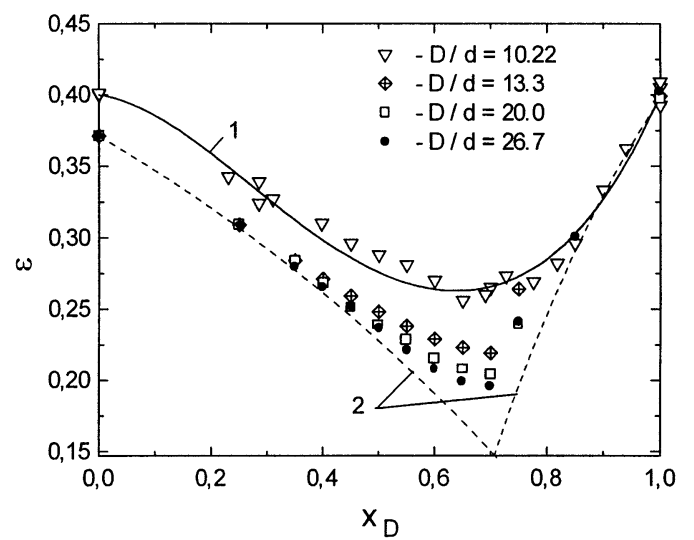

Figure 3. Measured $\epsilon$ for different $D / d$ values. For curve $1, D / d$ $=10.22, \epsilon_{D}^{0}=\epsilon_{d}^{0}=0.4$, and the model curve (from Mota et al. ${ }^{2}$ ). For curve 2 , the limit is defined by eqs 1 and $2(\delta \rightarrow 0)$ for $\epsilon_{d}^{0}=$ 0.371 and $\epsilon_{D}^{0}=0.4$.

average diameters of 2,3 , and $4 \mathrm{~mm}$ were obtained from Simax. The smaller bead sizes came from Sigmund Lindner: the first with an average diameter of 0.375 $\mathrm{mm}$, the second with an average diameter of $0.875 \mathrm{~mm}$, and the last one with an average diameter of $0.15 \mathrm{~mm}$. The particle density was $2500 \mathrm{~kg} / \mathrm{m}^{3}$ in every case.

Based on a preliminary investigation, it was found out that the most suitable method to be applied under the defined experimental conditions is similar to that described by Mills et al. ${ }^{43}$ Briefly, the procedure includes mixing the glass beads in the chosen proportion with a glycerol aqueous solution, filling the column, packing the column, and washing the glycerol out, followed by a checkup by means of image analysis of the particle fractions distribution in the column. The square column used in experiments had inner side dimensions of $5 \mathrm{~cm}$ and a height of $40 \mathrm{~cm}$. The square design was chosen to control, by automated image analysis, the homogeneity of the particle distribution. A previous work ${ }^{44}$ showed, by applying a $\chi^{2}$ test, that the square column used did not create wall effects. A square wire cloth from Haver \& Boecker, with an aperture width of $0.032 \mathrm{~mm}$, was used as layer support. The packing height in all the experiments remained at $10-15 \mathrm{~cm}$. Mixtures with a particle size ratio of $D / d=13.3,20$, and $26.7(\delta=$ $0.075,0.05$, and 0.0375 , respectively) were investigated. Experimentation with $D / d=10.22$ (i.e., $\delta=0.098$ ) was borrowed from a previous work. ${ }^{2}$ This means that four sets of experiments, covering the range of $x_{D}=0-1.0$, could be obtained for binary packed beds with $\delta<0.1$.

Porosity was always determined experimentally by filling the porous bed with water, which then was weighed. The experimental points shown in the figures below are the result of the average of three independent assays.

\section{Results and Discussion}

4.1. Porosity. The applied packing procedure allowed us to obtain a packing for small particles with $\epsilon_{d}^{0}=$ 0.371 ; however, for large-sized beads, the porosity was $\epsilon_{D}^{0}=0.4$. In Figure 3, the measured porosity of binary packing with $D / d=10.22,13.3,20$, and 26.7 (corresponding to $\delta=0.098,0.075,0.05$, and 0.0375 , respectively) is shown.

Experimental data show two types of porosity behavior: at the region $\delta \geq 0.1$, a smooth transition in the region of minimum porosity is observed. For lower $\delta$

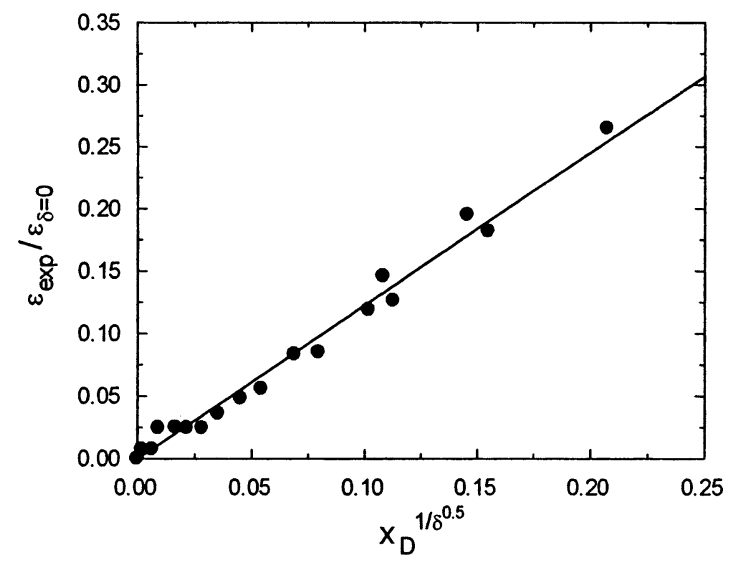

Figure 4. Dependence of $\ln \left(\epsilon_{\exp } / \epsilon_{\delta=0}\right)$ on $x_{D}{ }^{1 / \sqrt{\delta}}$ for $\epsilon_{d}^{0}=0.371$ and $D / d=13.3,20.0$, and 26.7. Points represent experimental data. Correlation coefficient is 0.993 .

values, the porosity trend follows the linear model. This behavior is observed in numerous works. ${ }^{4,8-10,12-19} \mathrm{It}$ is possible to characterize the region of minimum porosity as a transition of the filing mechanism, because, for $x_{D}$ $>0.4$, a skeleton of large particles is, more or less, filled with small particles. Furthermore, an internal wall effect in small particle arrangement around the large particles is noticeable for $x_{D}>0.4$. A well-known condition to obtain a homogeneous monosized packing in a vesse ${ }^{41,45-48}$ is that the ratio between particle size and the vessel diameter must be $>10$.

For small $\delta$, fractional porosities follow the linear model; however, in the range of minimum porosity, they are still far from the model prediction. In regard to $\epsilon_{D}$, the reason for deviation from boundary conditions is the wedging effect, whereas, for $\epsilon_{D}$, it is the interfractional wall effect.

For $\delta<0.1$, an approach similar to that used by Mota et al. $^{2}$ was used, i.e., a correction function was applied. In the ideal case, a correlation function may be based on eq 5 , considering the form $\epsilon=\epsilon\left(\epsilon_{d}^{0}, x_{D}\right) \cdot \varphi\left(\delta, x_{D}\right)$, where $\varphi\left(\delta, x_{D}\right)$ is a correction function. A linear dependence was obtained in the coordinates $\ln \left(\epsilon_{\exp } / \epsilon_{\delta=0}\right)$ vs $x_{D}{ }^{1 / \sqrt{\delta}}$ (Figure 4), where $\epsilon_{\exp }$ is the experimental porosity.

The best result was obtained for a correction function of the form

$$
\varphi\left(\delta, x_{D}\right)=\exp \left(1.2264 x_{D}^{1 / \sqrt{\delta}}\right)=\exp \left(1.2264 x_{D}^{\sqrt{D / d}}\right)
$$

Hence, the corrected equation will be

$$
\epsilon=\frac{\epsilon_{d}^{0}\left(1-x_{D}\right) \exp \left(1.2264 x_{D}^{1 / \sqrt{\delta}}\right)}{1-\epsilon_{d}^{0} x_{D}}
$$

which is valid for $x_{D} \leq x_{D, \min }$.

Application of the correction function (eq 13) gives good results (Figure 5), up to the minimum porosity composition. The model describes the porosity in the size ratio of the experiments well. When $\delta$ approaches zero $(\varphi(\delta) \rightarrow 1.0)$, the model creates the "ideal" curve that is given by eq 5 . The validity of this correction function can be accepted for $\delta \leq 0.1$.

4.2. Minimum Porosity. The porosity $\epsilon_{\min }$ decreases when the size ratio increases and converges to the 


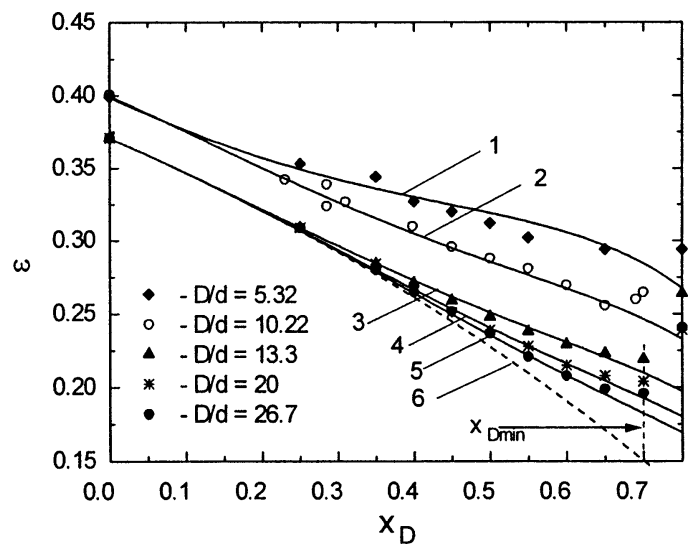

Figure 5. Comparison of the experimental porosity with the model defined in ref 12 : curve $1, D / d=5.32$; curve $2, D / d=10.22$; curve $3, D / d=13.3$; curve $4, D / d=20$; curve $5, D / d=26.7$; and curve 6 , data obtained using eq 5 .

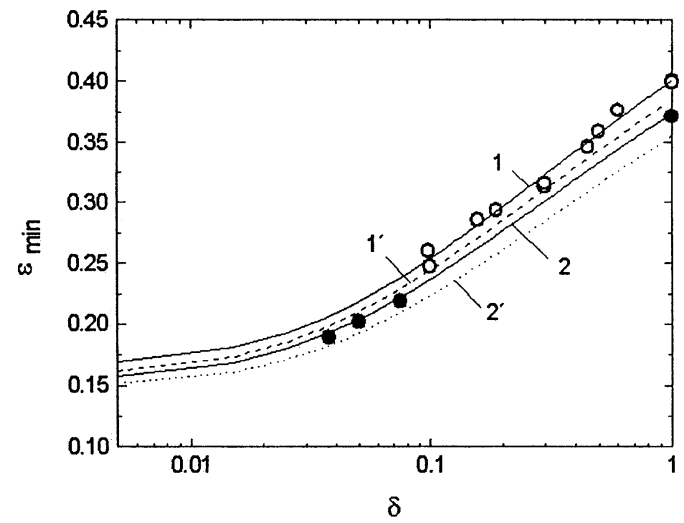

Figure 6. Dependence of $\epsilon_{\min }$ on $\delta$. Solid circles represent current measurements, and open circles represent data reported by Mota et al., ${ }^{2}$ curves represent data obtained using eq 15: 1,1', data obtained from Mota et al. ${ }^{2}$ (for curve $1, \epsilon_{D, s}^{0}=0.41, \epsilon_{d, s}^{0}=0.41$; for curve $1^{\prime}, \epsilon_{D}^{0}=0.4, \epsilon_{d}^{0}=0.4 ; 2,2^{\prime}$, current measurements (for curve $2, \epsilon_{D, s}^{0}=0.4, \epsilon_{d, s}^{0}=0.39$; for curve $2^{\prime}, \epsilon_{D}^{0}=0.4, \epsilon_{d}^{0}=0.371$ ).

absolute minimal value $\left(\epsilon_{\text {Min }}\right)$ for $D / d \rightarrow \infty$. When $D / d$ $\rightarrow \infty(\delta \rightarrow 0)$, the displacement or distortion effects of each particle fraction on fractional porosities becomes insignificant. Hence, the absolute minimal porosity is

$$
\epsilon_{\mathrm{Min}}=\epsilon_{D}{ }^{0} \epsilon_{d}{ }^{0}
$$

Because the packing procedure more or less induces a wall effect, the real average porosity of small particle packing at $x_{D, \min }$ is $\epsilon_{d, s}^{0} \geq \epsilon_{d}^{0}$. For the wedging effect, the large-particle skeleton porosity is $\epsilon_{D, s}^{0} \geq \epsilon_{D}^{0}$ and the minimum porosity is $\epsilon_{\min } \geq \epsilon_{\text {Min. }}$. Equation 14 can be used to estimate the minimum porosity at $x_{D \text {,min }}$, which is calculated from eq 8 by replacing $\epsilon_{D}^{0}$ with $\epsilon_{D, s}^{0}$ and by replacing $\epsilon_{d}^{0}$ with $\epsilon_{d, s}^{0}$ :

$$
\epsilon_{\min }=\frac{\epsilon_{d, s}^{0}\left(1-x_{D, \min }\right) \exp \left(1.2264 x_{D}{ }^{1 / \sqrt{\delta}}\right)}{1-\epsilon_{d, s}^{0} x_{D, \min }}
$$

Experimental values of the minimum porosity, in regard to previous measurements and current experiments, are shown in Figure 6 (open circles denote data for $\epsilon_{D}^{0}=0.4$ and $\epsilon_{d}^{0}=0.4$, from Mota et al., ${ }^{2}$ and solid circles represent data for $\epsilon_{D}^{0}=0.4$ and $\epsilon_{d}^{0}=0.371$ ), together with data calculated using eq 15 for different

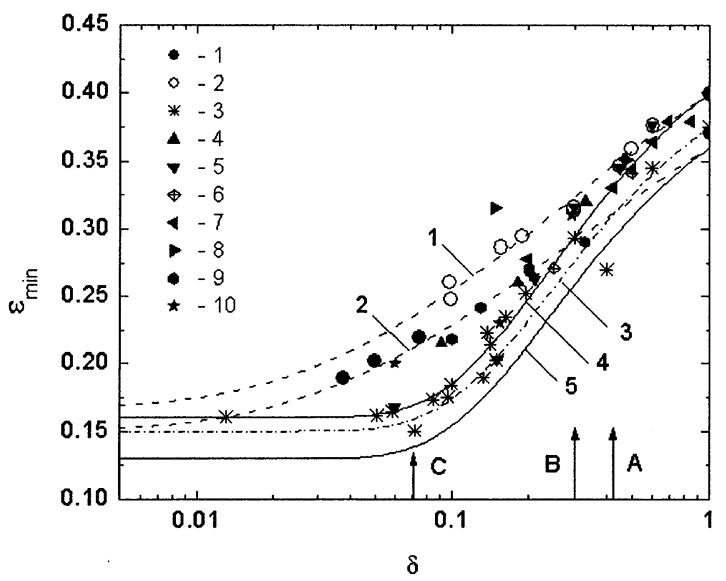

Figure 7. Dependence of $\epsilon_{\min }$ on $\delta$. Experimental data: 1, current measurements; 2, data from Mota et al. ${ }^{2} 3$, data from Liu and $\mathrm{Ha} ;{ }^{51}$ 4, data from Yu et al. ${ }^{50} 5$, data from Yu et al., ${ }^{13} 6$, data from Yu and Standish;17 7, data from MacDonald et al.; ${ }^{19}$ 8, data from Ouchiyama and Tanaka; ${ }^{34}$ 9, data from Yu and Standish ${ }^{24}$ and 10, data from Milewski. ${ }^{49}$ Curves: curve 1 , data obtained using eq 15 and $\epsilon_{D, s}^{0}=\epsilon_{d, s}^{0}=0.41$; curve 2 , data obtained using eq $15, \epsilon_{D, s}^{0}=0.4$, and $\epsilon_{d, s}^{0}=0.38$; curve 3 , data obtained using eq 16 for $\epsilon_{\text {Min }}=0.15$ and $\epsilon_{d}^{0}=0.375 ;{ }^{51}$ curve 4 , data obtained using eq $16, \epsilon_{\text {Min }}=0.16$, and $\epsilon_{d}^{0}=0.4 ;{ }^{51}$ and curve 5, data obtained using eq $16, \epsilon_{\text {Min }}=0.1296$, and $\epsilon_{d}^{0}=0.36 .^{51}$ (See text for an explanation of arrowed features A-C.)

conditions. To distinguish real fractional porosities $\left(\epsilon_{D, s}^{0}, \epsilon_{d, s}^{0}\right)$ from absolute porosities $\left(\epsilon_{D}^{0}, \epsilon_{d}^{0}\right)$, all values were used in eq 15 to obtain curves 1 and 2 and 1 ' and 2', respectively (see Figure 6).

As can be observed, eq 15 underestimates the minimum porosity of the experimental data when the absolute values of $\epsilon_{D}^{0}, \epsilon_{d}^{0}$ are used (see curves 1' and 2'). This fact leads to the conclusion that, even at $\delta=$ 0.0375 , the measured fractional porosity deviates from the values $\epsilon_{D}^{0}$ and $\epsilon_{d}^{0}$. It is useful to analyze, for dense and loose packing, the dependence of $\epsilon_{\min }$ on $\delta$ reported by several literature sources. Figure 7 represents a data collection that contains current research data, as well as data that has been gathered from different researchers, ${ }^{2,13,17,19,21,34,48-50}$ including the present authors.

It can be seen that the experimental data are rather divergent. Assuming that, for monosized packing $(\delta=$ $1.0)$, the porosity is likely to be in the range of $0.36-$ 0.4 , Figure 7 may help to establish bounds for $\epsilon_{\min }$ variation in cases of loose and dense packing, for a wide range of $\delta$. For loose packing, a good approximation is given by eq 15 (curves 1 and 2). For dense packing, the formula described in Liu and $\mathrm{Ha}^{51}$ and presented below,

$$
\epsilon_{\min }=\epsilon_{\mathrm{Min}}+\left(\epsilon_{d}^{0}-\epsilon_{\mathrm{Min}}\right) \exp \left[0.25\left(1-\frac{1}{\delta}\right)\right]
$$

was used, where $\epsilon_{\text {Min }}$ is represented by eq 15 .

In Figure 7, eq 16 was used for $\epsilon_{\text {Min }}=0.15$ and $\epsilon_{d}^{0}=$ 0.375 ; this plot is shown as curve 3. Assuming $\epsilon_{D}^{0}=\epsilon_{d}^{0}$ $=0.36-0.4$, a dense packing region that is bounded by curves 4 and 5 is obtained. The experimental data in Figure 7 clearly differentiate zones for $\delta$ related with specific packing mechanisms. One of the possible effects on the packing is the so-called wall effect. ${ }^{45}$ When tiny particles are close to large ones, large-sized particles act as a wall. The disturbance that is induced by the wall effect generated by the large particles may explain the 
overall porosity dependence on particle size ratio, as will be seen below.

4.3. Disturbance Effect of Small Particles. Usually, as Perry et al. reported, ${ }^{45}$ the wall effect propagates up to a distance of 4-5 sphere diameters from the wall. Pilotti, ${ }^{52}$ using a three-dimensional simulation of porous media of different-sized spherical particles, showed the presence of an additional void in the vicinity of the large particles. This surplus void dominates in the range of $\delta$ $=0.0667-0.333$, in accordance with data from Figure 7.

The distribution of glass particles around tubes was investigated by Schneider and Rippin, ${ }^{53}$ who showed that, when the distance between tubes decreases, porosity fluctuation increases with the increasing number of tubes. Accordingly, wall disturbances in binary mixtures will be dependent on the ratio of small particles $d$ to the pore throat diameter of the large particles skeleton $\left(D_{\text {th }}\right)$.

The ratio of small-sized particles $d$ to the pore throat diameter $D_{\text {th }}$ of the skeleton formed by large particles is $^{54}$

$$
\frac{d}{D_{\text {th }}}=\frac{\delta\left(1-\epsilon_{D}^{0}\right)}{0.62 \epsilon_{D}^{0}}
$$

The predominant porosity is $\epsilon_{D}^{0}=0.4$; therefore, all estimations made below will adopt this standard figure but may be easily extended to any other $\epsilon_{D}^{0}$ values.

The boundary condition for small particles to penetrate the large particle skeleton freely is $d / D_{\text {th }}=0.5$. At $d / D_{\text {th }}>0.5$, two beads coincide in crossing the same pore throat and complete filling is hindered. Putting this value into eq 17 results in $\delta \leq 0.207$. On the other hand, for $d / D_{\text {th }} \leq 0.25$, the size ratio becomes $\delta \leq 0.1$ (see arrowed features "B" and "C", respectively, in Figure 7).

For $0.25<d / D_{\text {th }}<0.5$, the effect of the void shape on small particles arrangement may be important and the filling mechanism might fail. The filling mechanism is dominant for $\delta<0.1$. This estimation correlates with the observations reported for systems that consisted of layers of different-sized particles. ${ }^{55}$

The following lines will be devoted to analyze the wall effect. Using a distance of 4-5 bead diameters from the wall, according to Perry et al., ${ }^{45}$ a cubic packing of large spherical particles $D$ is assumed and, for the porous media irregularity scale, a value of $x=5 d$ is observed. The ratio of solids volume to the skeleton void is equal to $\left(1-\epsilon_{D}^{0}\right) / \epsilon_{D}^{0} \approx 1.5$, where $\epsilon_{D}^{0}=0.4$. Replacing the void volume in the model with a cube of equal volume with a side dimension expressed by the scale of the irregularity zone $x$ gives $\pi D^{3} / 6=1.5(x m)^{3}=1.5(5 d m)^{3}$, or, by replacing $d / D$ with $\delta$,

$$
\delta=\frac{0.14}{m}
$$

where $m$ is the number of the scale units $(m \geq 2)$. For $m<2$, the irregularity zones are interfering with each other and the entire volume of small particles will be involved in the wall effect.

Because the fraction of nondisturbed length in the porous medium of small particle is $\eta=(m-2) / m$, or

$$
m=\frac{2}{1-\eta}
$$

from eq 19 follows the relation

$$
\delta=0.07(1-\eta)
$$

Adopting $\epsilon_{D}^{0}=0.36$, which is closer to the real packing value, the relationship given in eq 20 gives $\delta \approx 0.066(1$ $-\eta$ ). For $\epsilon_{D}^{0}=0.4$, values of $\delta$, as determined by eq 20 , are as follow: for $\eta=0.1, \delta=0.063$; for $\eta=0.5, \delta=$ 0.035 ; for $\eta=0.90, \delta=0.007$, and for $\eta=0.95, \delta=$ 0.0035. For a totally disturbed fraction, $\eta=0$ and the ratio is $\delta=0.07$. In turn, for $\delta<0.0035$, the wall effect becomes negligible $(\eta<0.95)$.

The remaining disturbing effect on packed bedswedging-will now be examined. Wedging is likely to occur whenever packed beds need to be washed or regenerated (e.g., ion-exchange resins). The countercurrent flow expands or fluidizes the bed and rearrangements occur. The limit situation occurs when every single large particle is kept apart from its neighbors, being sustained in that position by a minimum number of small beads. The necessary and sufficient number of small beads is the coordination number of the packing. For a cubic packing, the coordination number is $N_{\mathrm{c}}=$ 6 . Therefore, the increase in the void volume will be achieved by only six small beads, with a negligible solid volume. The volume after wedging will be

$$
V^{\prime}=\frac{\pi}{6}(D+2 \delta D)^{3}
$$

After neglecting the higher-order terms that result from the development of the cubic expression, the following equation is obtained:

$$
V^{\prime}=\left(\pi D^{3}\right)\left(\frac{1+5 \delta}{6}\right)
$$

The ratio between the new volume and the original volume will be

$$
\frac{V^{\prime}}{V}=1+5 \delta
$$

The increase in porosity fraction due to wedging will be, at most, $5 \delta$. Table 2 displays a set of results for different $\delta$ values.

It is unlikely that this increase occurs to this extent, because it would mean that small beads would be segregated from the packing. It is worthy of note that wedging is probably less disturbing than the wall effect, because the latter starts to be significant-more than $5 \%$ disturbance-from $\delta \geq 0.0035$. It also is interesting to note that, in the range of $0.01 \leq \delta \leq 0.07$, both mechanisms have a significant impact on the porosity of the medium.

\section{Conclusion}

The arrangement of large- and small-sized particles in a binary mixture is determinant for the overall porosity: small particles affect the large particles by

Table 2. Maximum Increase in Porosity by Wedging for Different Size Ratios

\begin{tabular}{cc}
\hline$\delta$ & increase in porosity $(\%)$ \\
\hline 0.01 & 5 \\
0.02 & 10 \\
0.035 & 17.5 \\
0.1 & 50
\end{tabular}


displacement and wedging, whereas large particles affect the small particles by the wall effect near their own surface. The outcome of mentioned effects is dependent on the packing procedure that will, in turn, result in the different correction functions used in numerous models.

According to the estimations given above, five regions on the dependence of $\epsilon_{\min }$ versus $\delta$ can be identified:

(1) A region of $\delta>0.41$, where a displacement mechanism occurs (see arrowed feature marked "A" in Figure 7).

(2) A region where the linear mixing model is valid $(\delta \approx 0.2$; see arrowed feature marked "B" in Figure 7 ).

(3) A region where small particles completely fill the skeleton void $(0.01<\delta<0.2$; see arrowed feature marked "C" in Figure 7). In this region, the wall effect and wedging might occur at the same time. This phenomenon may occur during the washing and regeneration of chromatographic and ion-exchange columns.

(4) A region of small particles arrangement, where $0.0035<\delta<0.01$. In this case, there will be no significant wedging, but the wall effect is still disturbing.

(5) A region of small particles arrangement, where $\delta$ $<0.0035$. In this case, neither the wall effect nor wedging are significant and the small particles can totally invade the internal void space between large particles.

The developed approach is useful to understand binary mixtures and shows that the approach based on the fractional porosity $\epsilon_{D}\left(x_{D}\right)$ and $\epsilon_{d}\left(x_{D}\right)$ may be a powerful tool to control the overall porosity, giving a new insight on mixture structure and reasonable explanations for the different types of the porosity behavior in the region of the minimum porosity.

\section{Acknowledgment}

The authors acknowledge the grant that was given to A.I.Y. by Fundação para a Ciência e Tecnologia (FCT), without which this work would not be possible. The authors wish also to acknowledge the FCT funding of Project POCTI/EQU/37500/2001, under which the present research was performed. This project was partially funded by FEDER.

\section{Nomenclature}

Symbols

$a=$ correction coefficient for $\epsilon_{d}^{0}$ in the binary packing

$D=$ diameter of large particles $(\mathrm{m})$

$d=$ diameter of small particles $(\mathrm{m})$

$d_{\text {av }}=$ average particle diameter in the mixture (m)

$D_{\text {por }}=$ pore diameter $(\mathrm{m})$

$D_{\text {th }}=$ throat diameter $(\mathrm{m})$

$L=$ bed thickness $(\mathrm{m})$

$L_{\mathrm{e}}=$ average pathway length in the bed (m)

$m=$ number of the scale units to induce disturbance wall effect

$x=$ irregularity scale

$x_{D}=$ volume fraction of large particles in the total volume of particles in the mixture

$x_{D \text {,min }}=$ volume fraction of large particles corresponds to the minimum mixture porosity

Greek Letters

$\epsilon=$ overall porosity of a mixed bed

$\epsilon_{D}=$ fractional porosity of the large-sized particle fraction $\epsilon_{d}=$ fractional porosity of the small-sized particle fraction

$\epsilon_{\text {Min }}=$ absolute minimum porosity

$\epsilon_{\min }=$ minimal porosity of a mixed bed

$\epsilon_{D}^{0}=$ porosity of a uniform bed of large particles

$\epsilon_{d}^{0}=$ porosity of a uniform bed of small particles

$\epsilon_{D, s}^{0}=$ large-particle skeleton porosity at the point of minimum porosity in the case of a

wedging effect

$\epsilon_{d, s}^{0}=$ small-sized particle packing porosity in the skeleton void

$\delta=$ particle size ratio, $\delta=d / D$

$\eta=$ fraction of nondisturbed length of the porous medium, $\eta=(m-2) / m$

\section{Literature Cited}

(1) Ben Aïm, R.; Le Goff, P.; Le Lec, P. La Perméabilité de Milieux Poreux Formés par Empilement de Mélanges Binaires de Grains Sphériques. Powder Technol. 1971, 5 (1), 51-60.

(2) Mota, M.; Teixeira, J. A.; Yelshin, A. Binary Spherical Particle Mixed Beds Porosity and Permeability Relationship Measurement. Trans. Filtr. Soc, 2001, 1 (4), 101-106.

(3) Shahinpoor, M. Statistical Mechanical Considerations on the Random Packing of Granular Materials. Powder Technol. 1980, 25 (2), 163-176.

(4) Suzuki, M.; Makino, K.; Yamada, M.; Iinoya, K. A Study on the Coordination Number in a System of Randomly Packed, Uniform-Sized Spherical Particles. Int. Chem. Eng. 1981, 21 (3), $482-488$.

(5) Greenkorn, R. A. Steady Flow through Porous Media. AIChE J. 1981, 27 (4), 529-543.

(6) Huizenga, D. G.; Smith, D. M. Knudsen Diffusion in Random Assemblages of Uniform Spheres. AIChE J. 1986, 32 (1), $1-6$.

(7) Wright, T.; Smith, D. M.; Stermer, D. L. Knudsen Diffusion in Bidisperse Mixtures of Uniform Spheres. Ind. Eng. Chem. Res. 1987, 26 (6), 1227-1232.

(8) Jeschar, R. Druckverlust in Mehrkornschüttungen aus Kugeln. Arch. Eisenhüttenwes. 1964, 35, 91-108.

(9) Ben Aïm, R.; Le Goff, P. Effet de Paroi dans les Empilements Désordonnés de Sphères et Application à la Porosité de Mélanges Binaires. Powder Technol. 1968, 1 (5), 281-290.

(10) Brauer, H. Grundlagen der Einphasen-und Mehrphasenströmungen; Verlag Sauerländer: Aarau, Germany, 1971.

(11) Ouchiyama, N.; Tanaka, T. Porosity of Mass of Solid Particles Having a Range of Sizes. Ind. Eng. Chem. Fundam. 1981, 20 (1), 66-71.

(12) Le Goff, P.; Leclerc, D.; Dodds, J. The Structure of Packed Beds: Continuity of Research in Nancy and Some New Results. Powder Technol. 1985, 42, 47-53.

(13) Yu, A. B.; Zou, R. P.; Standish, N. Modifying the Linear Packing Model for Predicting the Porosity of Nonspherical Particle Mixtures. Ind. Eng. Chem. Res. 1996, 35 (10), 3730-3741.

(14) Hulewicz, Z. Z. Resistance to Flow through a Granular Bed in the Laminar Regime. I. Derivation of a New Correlating Equation. Int. Chem. Eng. 1987, 27 (3), 566-573.

(15) Gotoh, K.; Chuba, T.; Suzuki, A. Computer Simulation of Weight Distributions of Spherical-Particle Beds on the Bottom of a Container. Int. Chem. Eng. 1982, 22 (1), 107-115.

(16) Kuramae, M. Investigation of Unsaturated Liquid Flow in a Granular Bed Using a Cubic-Lattice Pore Model. Int. Chem. Eng. 1982, 22 (4), 666-673.

(17) $\mathrm{Yu}, \mathrm{A} . \mathrm{B}$; Standish, N. Estimation of the Porosity of Particle Mixtures by a Linear-Mixture Packing Model. Ind. Eng. Chem. Res. 1991, 30 (6), 1372-1385.

(18) Macé, O.; Wei, J. Diffusion in Random Particle Models for Hydrodemetalation Catalysts. Ind. Eng. Chem. Res. 1991, 30 (5), 909-918.

(19) MacDonald, M. J.; Chu, C.-F.; Pierre, P. P.; Ng, K. M. A Generalized Blake-Kozeny Equation for Multisized Spherical Particles. AIChE J. 1991, 37 (10), 1583-1588.

(20) Mota, M.; Teixeira, J. A.; Yelshin, A.; Bowen, W. R Interference of Coarse and Fine Particles of Different Shape in Mixed Porous Beds and Filter Cakes. Miner. Eng. 2003, 16 (2), 135-144

(21) Mota, M.; Teixeira, J. A.; Bowen, R.; Yelshin, A. Effect of Tortuosity on Transport Properties of Mixed Granular Beds. In 
Proceedings of 8th World Filtration Congress, April 3-7, 2000; Filtration Society: Brighton, U.K., 2000; Vol. 1, pp 57-60.

(22) Abe, E.; Hirosue, H. Porosity Estimation of a Mixed Cake in Body Filtration. J. Chem. Eng. Jpn. 1982, 15 (6), 490-493.

(23) Yu, A. B.; Bridgwater, J.; Burbidge, A. On the Modelling of the Packing of Fine Particles. Powder Technol. 1997, 92, 185194.

(24) Yu, A. B.; Standish, N. A Study of the Packing of Particles with a Mixture Size Distribution. Powder Technol. 1993, 76, 113124.

(25) Mathias, M. F.; Muldowney, G. P. Effect of Solids Loading Method on the Bed Porosity and Gas Flux Distribution in a FixedBed Reactor. Chem. Eng. Sci. 2000, 55 (21), 4981-4991.

(26) Zhang, Z. P.; Yu, A. B.; Oakeshott, R. B. S. Effect of Packing Method on the Randomness of Disc Packings. J. Phys. A: Math. Gen. 1996, 29, 2671-2685.

(27) Wightman, C.; Mort, P. R.; Muzzio, F. J.; Riman, R. E.; Gleason, E. K. The Structure of Mixtures of Particles Generated by Time-Dependent Flows. Powder Technol. 1995, 84 (3), 231240

(28) Al-Dahhan, M. H.; Wu, Y.; Dudukovic, M. P. Reproducible Technique for Packing Laboratory-Scale Trickle-Bed Reactors with a Mixture of Catalyst and Fines. Ind. Eng. Chem. Res. 1995, 34 (3), 741-747.

(29) Zou, R. P.; Yu, A. B. Evaluation of the Packing Characteristics of Mono-sized Nonspherical Particles. Powder Technol. 1996, 88, 71-79.

(30) Caglioti, E.; Coniglio, A.; Herrmann, H. J.; Loreto, V.; Nicodemi, M. Geometrical Frustration: A Dynamical Motor for Dry Granular Media. Physica A 1998, 257, 419-423.

(31) Nicodemi, M. A Phenomenological Theory of Dynamic Processes in Granular Media. Physica A 1998, 257, 448-453.

(32) Caglioti, E.; Loreto, V.; Herrmann, H. J.; Nicodemi, M. A "Tetris-like" Model for the Compaction of Dry Granular Media. Phys. Rev. Lett. 1997, 79 (8), 1575-1578.

(33) Nicodemi, M.; Coniglio, A.; Herrmann, H. J. Frustration and Slow Dynamics of Granular Packings. Phys. Rev. E 1997, 55 (4), 3962-3969.

(34) Ouchiyama, N.; Tanaka, T. Porosity Estimation for Random Packings of Spherical Particles. Ind. Eng. Chem. Fundam. 1984, 23 (4), 490-493.

(35) Yu, A. B.; Standish, N.; McLean, A. Porosity Calculation of Binary Mixtures of Nonspherical Particles. J. Am. Ceram. Soc. 1993, 76 (11), 2813-2816.

(36) Finkers, H. J.; Hoffmann, A. C. Structural Ratio for Predicting the Voidage of Binary Particle Mixtures. AIChE J. 1998, 44 (2), 495-498.

(37) Bear, J.; Bachmat, Y. Transport Phenomena in Porous Media-Basic Equations. In Fundamentals of Transport Phenomena in Porous Media; Bear, J., Corapcioglu, M. Y., Eds.; NATO ASI Series E: Applied Sciences 82; Martinus Nijhoff: Dordrecht, The Netherlands, 1984; pp 3-61.

(38) Lessmann, R. C. A Conceptual Framework for Predicting the Filtration Performance of Nonwoven Fibrous Filter Media. Fluid/Part. Sep. J. 1988, 1 (6), 35-43.
Ind. Eng. Chem. Res., Vol. 43, No. 24, 2004

(39) Govindarao, V. M. H.; Froment, G. F. Voidage Profiles in Packing Beds of Spheres. Chem. Eng. Sci. 1986, 41 (3), 533-539.

(40) Scott, G. D. Packing of Spheres. Nature 1960, 188 (10), 908-909.

(41) Zou, R. P.; Yu, A. B. The Packing of Spheres in a Cylindrical Container: The Thickness Effect. Chem. Eng. Sci. 1995, 50 (9), 1504-1507.

(42) Mota, M.; Teixeira, J. A.; Yelshin, A. Image Analysis of Packed Beds of Spherical Particles of Different Sizes. Sep. Purif. Technol. 1999, 15 (15), 59-68.

(43) Mills, P. J. T.; Seville, J. P. K.; Knight, P. C.; Adams, M. J. The Effect of Binder Viscosity on Particle Agglomeration in a Low Shear Mixer/Agglomerator. Powder Technol. 2000, 113 (12), 140-147

(44) Dias, R.; Teixeira, J. A.; Mota, M.; Yelshin, A. Preparation of Controlled Particulate Mixtures with Glass Beads of Different Sizes. Sep. Purif. Technol. 2004, 37 (1), 69-80.

(45) Perry, R. H.; Green, D. W.; Maloney, J. O. Perry's Chemical Engineers Handbook, 6th ed.; McGraw-Hill: New York, 1988.

(46) Sederman, A. J.; Alexander, P.; Gladden, L. F. Structure of Packed Beds Probed by Magnetic Resonance Imaging. Powder Technol. 2001, 117 (3), 255-269.

(47) Zou, R. P.; Yu, A. B. Wall Effect on the Packing of Cylindrical Particles. Chem. Eng. Sci. 1996, 51 (7), 1177-1180.

(48) McWhirter, J. D.; Crawford, M. E.; Klein, D. E. Wall Region Porosity Distributions for Packed Beds of Uniform Spheres with Modified and Unmodified Walls. Transp. Porous Media 1997, 27, 99-118.

(49) Milewski, J. V. The Combined Packing of Rods and Spheres in Reinforcing Plastics. Ind. Eng. Chem. Prod. Res. Dev. 1978, 17 (4), 363-366

(50) Yu, A. B.; Zou, R. P.; Standish, N. Packing of Ternary Mixtures of Nonspherical Particles. J. Am. Ceram. Soc. 1992, 75 , $2765-2772$.

(51) Liu, S.; Ha, Z. Prediction of Random Packing Limit for Multimodal Particle Mixtures. Powder Technol. 2002, 126, 283296.

(52) Pilotti, M. Generation of Realistic Porous Media by Grains Sedimentation. Transp. Porous Media 1998, 33, 257-278.

(53) Schneider, F. A.; Rippin, D. W. T. Determination of the Local Voidage Distribution in Random Packed Beds of Complex Geometry. Ind. Eng. Chem. Res. 1988, 27 (10), 1936-1941.

(54) Aerov, M.; Todes, O.; Narinskii, D. Apparatuses with Stationary Granular Bed; Khimia: Leningrad, Russia, 1979.

(55) Propster, M.; Szekely, J. The Porosity of Systems Consisting of Layers of Different Particles. Powder Technol. 1977, 17 (1), $123-128$.

Received for review February 6, 2004 Revised manuscript received August 30, 2004 Accepted September 3, 2004

IE040048B 